\title{
Observation of the nonlinear phase shift due to single post-selected photons
}

\author{
Amir Feizpour ${ }^{1 \star}$, Matin Hallaji ${ }^{1}$, Greg Dmochowski ${ }^{1}$ and Aephraim M. Steinberg ${ }^{1,2}$
}

Over the past years, much effort has gone towards generating interactions between two optical beams so strong that they could be observed at the level of individual photons ${ }^{1-3}$. Interactions this strong, beyond opening up a new regime in optics ${ }^{4}$, could lead to technologies such as all-optical quantum information processing ${ }^{5,6}$. However, the extreme weakness of photon-photon scattering has hindered any attempt to observe such interactions at the level of single particles. Here we present an implementation of a strong optical nonlinearity using electromagnetically induced transparency ${ }^{7}$, and a direct measurement of the resulting nonlinear phase shift for single post-selected photons. We show that the observed phase shift depends not only on the incident intensity of the (coherent-state) input signal, but also in a discrete fashion on whether 0 or 1 photons are detected at the output. We believe that this constitutes the first direct measurement of the cross-phase shift due to single photons, whose presence or absence is established based on a discrete detection event. It opens a door to future studies of nonlinear optics in the quantum regime, and potential applications in areas such as quantum information processing.

Modern optical physics has revolved principally about two poles: nonlinear optics, where rich effects are generated through the interactions of photons with one another, but which because of the weakness of those interactions typically manifests itself only for pulses containing billions of photons; and quantum optics, where phenomena such as entanglement have been widely studied, but where photon-photon interactions are negligible. In fact, it has been well known in quantum optics since the Nobel Prize-winning work of R. Glauber that, in the linear regime, the classical and quantum theories of electromagnetism make identical predictions (except in the presence of nonclassical sources of light-which themselves rely on nonlinear effects) $)^{8,9}$. It has therefore long been a dream to move into the realm of 'quantum nonlinear optics', where sufficiently strong interactions could create a complex many-body interacting quantum system in the context of optics. Such interactions could enable the production and detection of novel entangled states, including few-photon bound states ${ }^{4,10}$, as well as new architectures for non-demolition measurement of photon number ${ }^{11-13}$, quantum teleportation ${ }^{14}$, lowlight-level switching ${ }^{3}$ and quantum logic gates ${ }^{5}$. Decades ago, important steps in this direction were taken in the realm of cavity quantum electrodynamics (QED) (ref. 15), leading for instance to major advances in the study of entanglement ${ }^{16-19}$, and more recent significant developments in the context of superconducting qubits $^{20,21}$. Greatly enhanced interactions for photons have recently been observed, using electromagnetically induced transparency and slow light ${ }^{1}$, microstructured fibres ${ }^{22}$, atoms in hollow fibres ${ }^{23}$, a single atom strongly coupled to a micro-resonator cavity ${ }^{24}$, atoms or other impurities near tapered fibres ${ }^{25,26}$ or bottle resonators ${ }^{27}$. Most recently, the application of 'Rydberg blockades ${ }^{28-30}$ has enabled huge interaction strengths, already leading to the observation of strongly modified quantum statistics ${ }^{10,31-35}$. Sum-frequency generation of two heralded single photons has also been demonstrated ${ }^{36}$. In parallel, there has been some controversy about the applicability of such strong interactions to quantum logic due to fundamental noise limits $^{37-39}$ and suggestions that intermediate-strength nonlinearities might be a powerful approach ${ }^{6,40}$. These proposals put forward a way to use interactions between single photons and probe pulses containing many photons to implement a near-deterministic controlled-phase gate. The requirement for these schemes is simply to have a detectable cross-phase shift (XPS) imparted by a single photon on a classical beam, rather than a nonlinear phase shift of the order of $\pi$ radians as in the earlier proposals. At the present time, however, the nonlinear phase shift written by a single photon on a probe beam has to our knowledge never been reported, and previous experiments have been performed with classical pulses with average photon numbers of the order of a few hundred $^{41}$ in free space, or as low as 16 or 0.1 in a hollow-core fibre filled with atomic vapour ${ }^{23}$ or a nonlinear photonic-crystal fibre $^{22}$, respectively.

Here we demonstrate that by illuminating a sample of atoms with a weak coherent state, but post-selecting on subsequent detection of a photon at the far side of the sample, we can observe the nonlinear effect of that one additional photon on a probe beam. Similar 'intensity-field correlations' were previously used to enhance quantum effects in a cavity-QED system ${ }^{42-44}$. Using electromagnetically induced transparency in a gas of lasercooled ${ }^{85} \mathrm{Rb}$ atoms, we generate a nonlinear phase shift of $18 \mu \mathrm{rad}$ per photon, and unequivocably demonstrate the contribution due to individual post-selected photons. The sample was illuminated with weak coherent-state pulses with as few as 0.5 photons on average, and post-selection allowed us to subsequently determine whether a given pulse contained (approximately) 0 or 1 photons. Our data unambiguously exhibit a discrete dependence of the observed phase shift on the post-selection outcome. This represents the first direct measurement of the XPS due to a post-selected single photon.

We use an atomic level scheme ${ }^{1}$ based on electromagnetically induced transparency ${ }^{7}$ (EIT), which allows very strong nearresonant interactions. EIT is a coherent atomic effect in which different excitation pathways interfere destructively, eliminating linear absorption and simultaneously producing sharp dispersive slopes; see the inset of Fig. 1. To establish EIT, two phase-coherent

${ }^{1}$ Centre for Quantum Information and Quantum Control and Institute for Optical Sciences, Department of Physics, University of Toronto, 60 St George Street, Toronto, Ontario M5S 1A7, Canada. ${ }^{2}$ Canadian Institute for Advanced Research, 180 Dundas St W., Toronto, Ontario M5G 1Z8, Canada. *e-mail: feizpour@physics.utoronto.ca 


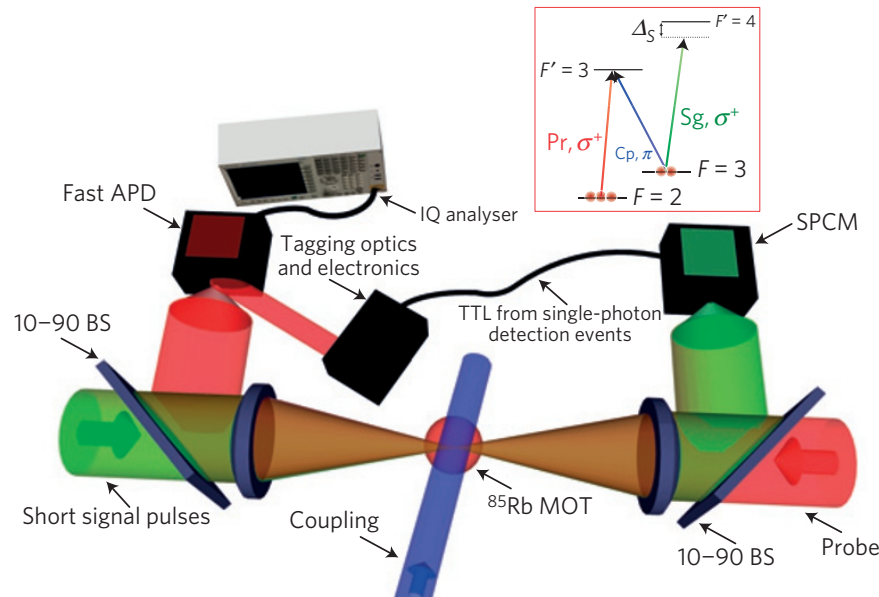

Figure 1 | Schematic of the experimental set-up. Counter-propagating probe and signal beams are focused to a waist of $13 \pm 1 \mu \mathrm{m}$ inside a cloud of laser-cooled ${ }^{85} \mathrm{Rb}$ atoms confined in a magneto-optical trap (MOT). High reflectivity beamsplitters (BS; $90 \%$ reflectivity) are used to collect the signal and probe beams after the interaction. A collimated coupling beam, propagating perpendicular to both probe and signal beams, creates a $2 \mathrm{MHz}$ EIT window for the probe (see the inset). The amplitude and phase of the probe are measured using frequency-domain interferometry. The single-photon detection events on the SPCM are registered as tags in the amplitude of the probe. The inset shows the level scheme used.

laser fields (probe and coupling) form a $\Lambda$-system, addressing a common excited state. When the two-photon resonance condition is satisfied — that is, their respective detunings are equal—the medium becomes transparent to these fields and each experiences a modified refractive index profile. The presence of an additional 'signal' field inside the medium serves to a.c.-Stark shift one of the ground states of the $\Lambda$-system, pulling the probe and coupling fields out of twophoton resonance. As a result, the probe field experiences a change in refractive index, acquiring a phase shift that is proportional to the slope of this steep dispersive feature. For low signal power, the induced Stark shift is linear in signal intensity; the resulting probe phase shift is, therefore, proportional to the number of photons in the signal pulse. Measurement of this phase shift provides information about the number of signal photons present in the interaction region.

Figure 1 shows a schematic of the experimental set-up as well as the level scheme. A cloud of laser-cooled ${ }^{85} \mathrm{Rb}$ atoms serves as the nonlinear optical medium, with the probe and coupling beams passing through orthogonal to each other. Short signal pulses are sent counter-propagating to the probe and both beams are collected by highly reflective beamsplitters after the interaction. A singlephoton counting module (SPCM) is used to detect the signal pulses whereas the probe beam is detected by a fast avalanche photodiode (APD), operating in the linear regime; see the 'Methods' section for the details of the probe phase measurement.

We first measure the XPS for a range of signal pulse energies to determine the size of the per-photon effect. Figure 2 plots the XPS on the probe field versus the average number of photons per signal pulse. The phase shift grows linearly for low photon numbers and a fit yields a slope of $13 \pm 1 \mu \mathrm{rad}$ per photon. There is a saturation at high photon numbers that occurs when the induced a.c.-Stark shift becomes comparable to or larger than the half-width of the EIT window. The lowest energy per pulse that we use here corresponds to an average of one photon per pulse, which is the lowest pulse energy ever used for cross-phase modulation in free space.

Although in the measurement described above we have observed the XPS due to signal pulses with an average photon number of 1 , approximately $40 \%$ of the pulses contain no photons at all; and about

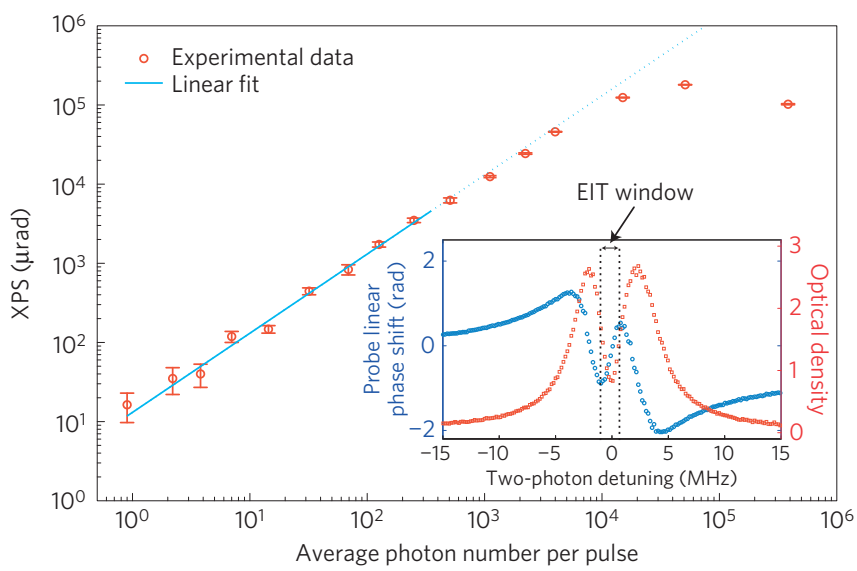

Figure 2 | XPS versus average photon number per pulse. The nonlinear phase shift depends linearly on the photon number at lower intensities. A fit to the low-photon-number data yields a slope of $13 \pm 1 \mu$ rad per photon whereas the deviation at higher photon numbers arises due to higher-order nonlinearities. The signal centre detuning is $-10 \mathrm{MHz}$, the on-resonance OD in the absence of the coupling is 2 , and the EIT window width is $2 \mathrm{MHz}$. The inset shows a typical linear phase profile (blue circles) and optical density (red squares) as seen by the probe. The vertical lines indicate our definition of the EIT window throughout this paper (namely, the full-width at half-maximum of the transparency. The error bars represent the standard deviation of the mean.

$25 \%$ contain multiple photons. To observe the quantized effect of individual photons, we send in even weaker pulses-containing only one-half a photon on average-and trigger on subsequent detection of a single photon at an SPCM. These so-called 'click' events occur only a small fraction of the time, due to the weak signal pulses as well as detector inefficiency. Because of this finite efficiency, the

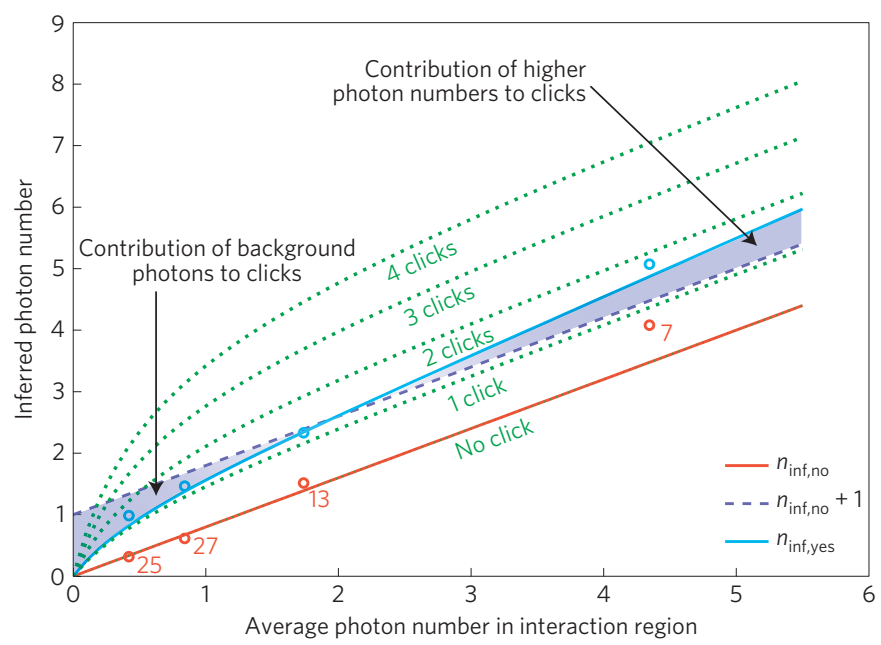

Figure 3 | Inferred $\left(n_{\text {inf }}\right.$ ) versus average photon number in the interaction region. The overall collection efficiency is assumed to be $20 \%$ and the background click rate is taken to be $10 \%$ for solid and dotted lines. The circles show the photon number values inferred for the data points in Fig. 4 for no-click (red) and click (blue) events. The inferred photon numbers depend on the overall detection deficiency, $\eta$. As we varied this efficiency for different data points, we indicate it (in \%) next to each circle. The dotted green lines show the photon number which would be inferred were a number-resolving detector used. The solid blue line could also be obtained from a weighted average of the dotted lines with non-zero number of clicks. The dashed blue line shows where the difference in the inferred photon number for click- and no-click events is equal to 1. 


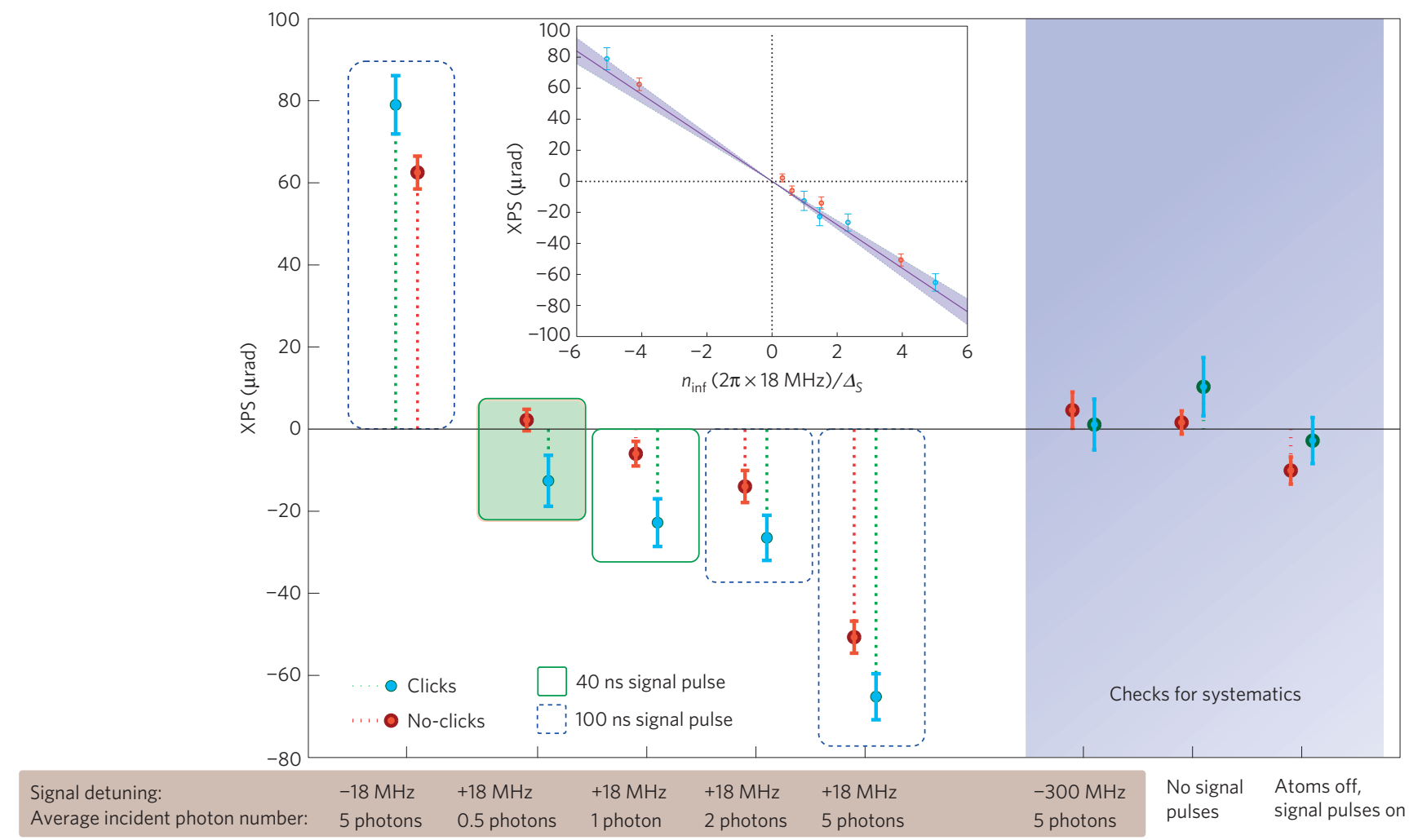

Figure 4 | Post-selected single-photon XPS. Most notably, for an average incident photon number of 0.5 (green-shaded region), the XPS for no-click and click events are $2 \pm 3$ and $-13 \pm 6 \mu \mathrm{rad}$, respectively, which definitively shows the effect of a single post-selected photon. For the other data points, the average incident photon number and/or the signal centre detuning is varied. Taking all the data points together, the magnitude of the post-selected single-photon XPS is $-18 \pm 4 \mu \mathrm{rad}$. The inset shows the post-selected XPS versus $n_{\text {inf }}(2 \pi \times 18 \mathrm{MHz}) / \Delta_{s}$, inferred average photon number corrected for the variable signal detuning. The solid line has a slope of $-14 \pm 1 \mu$ rad per $18 \mathrm{MHz}$-detuned photon. The EIT window width is $2 \mathrm{MHz}$ and the on-resonance OD in the absence of the coupling is 3 . The data in the region shaded in blue are tests for systematics, as explained in the text. The error bars represent the standard deviation of the mean.

absence of a click does not preclude the possibility that there had, in fact, been a photon in the interaction region. Nevertheless-and neglecting for the moment background counts and multi-photon events-it can be shown that the best estimate possible of the photon number in the interaction region increases by exactly one when the detector fires, regardless of its efficiency. An intuitive way to understand this is via the common model of an inefficient detector as equivalent to a beamsplitter, separating 'detected' and 'undetected' modes. Splitting a quasi-classical (coherent) state into two modes generates a product state with no correlations; thus the best estimate of the number of undetected photons is independent of how many detected photons are observed. Therefore, when one 'click' event occurs, our estimate of the total photon number is greater (by that one photon) than when no click occurs. Comparing cases when the SPCM fires with cases where it does not thus provides a measure of the effect of a single additional photon.

When background and multi-photon events are included, one can still calculate the shift in inferred average photon number due to a 'click' event, and it remains close to (but no longer exactly equal to) 1 ; see the Supplementary Information for details. This difference depends on the incident average photon number, $|\alpha|^{2}$, the overall collection and detection efficiency, $\eta$, and the background photon detection probability, $P_{\mathrm{b}}$. When the probability of clicks due to signal photons is much higher than those due to background photonsbut still much lower than $1\left(P_{\mathrm{b}} \ll \eta|\alpha|^{2} \ll 1\right)$-the difference is close to 1 . However, at low incident photon numbers false clicks due to the background photons cause the inferred difference to be smaller than 1. On the other hand, at high incident photon numbers, the difference can be larger than 1 owing to the contribution of multiphoton events to the single-click measurements (when non-photonresolving detectors are used). Figure 3 plots the inferred average photon number in the interaction region, $n_{\text {inf }}$, versus the average incident photon number, $|\alpha|^{2}$, for click and no-click events. In this figure, the circles show the inferred photon number for the parameters used in the experiment. The average photon numbers in the interaction region for these data points are lower than the corresponding incident photon numbers because of the finite signal absorption (75\% transmission).

We can separate out the instances in which the SPCM detected a photon (the 'click' cases) from those in which no click was registered by the detector. This allows us to measure the nonlinear phase shift of the probe in the two cases separately (see Fig. 4). For 0.5 incident signal photons per pulse, the inferred average photon number in the interaction region for no-click events is roughly 0.3 , whereas it is approximately 1 for click events; see the square shaded in green. The phase shift measured for the no-click cases is statistically indistinguishable from zero $(2 \pm 3 \mu \mathrm{rad})$, whereas the click events result in a non-zero phase shift of $-13 \pm 6 \mu \mathrm{rad}$, consistent with the per-photon nonlinear phase shift of $-13 \pm 1 \mu \mathrm{rad}$ inferred from the fit to the data in Fig. 2.

For the remaining data points, the average incident signal photon number and/or the centre detuning of signal pulses was varied. The most significant feature is that the nonlinear phase shifts for click events are always larger in absolute size than those for the no-click cases. The magnitude of the phase difference between click and no-click events averaged over all data points-that is, 
the nonlinear phase shift due to a post-selected single photon-is $-15 \pm 3 \mu \mathrm{rad}(-18 \pm 4 \mu \mathrm{rad}$ after correcting for finite background; see the Supplementary Information for details).

To confirm that the observed effect is not due to systematics, we have also taken data in the absence of signal pulses or atoms, or with large signal detunings. The data in the region shaded in blue illustrates these checks for systematics. The most important feature to highlight is that the click- and no-click probe phase shifts for all systematic checks are equal to within the error bars.

The post-selected XPS data has a linear dependence on inferred average photon number. The inset of Fig. 4 plots all the data points versus inferred average photon number corrected for the variable signal detuning (see Supplementary Information). The data agree well with the XPS per photon expected from our earlier measurements corrected for signal detuning and different optical densities, shown as a solid line.

The theoretical prediction for the magnitude of the XPS, given the parameters of our experiment, is 13 urad based on the model presented in ref. 45 (see Supplementary Information for more details). The values we measured in our experiment are in very good agreement with the theoretical value.

There are a number of important directions for extensions to this work. One is to repeat the experiment with signal light with different quantum statistics, in which case the dependence of phase shift on detection events will change; in particular, for bunched light, a larger phase shift per detection event is to be expected. With anti-bunched sources ${ }^{46}$, of course, post-selection will no longer be required. Moving into the regime of single-shot resolvability required for quantum logic gates based on weak optical nonlinearities ${ }^{6,40}$ will require using a tighter beam focus, higher optical density, and narrower EIT windows, most likely combined with more sophisticated level schemes, such as ones in which both probe and signal beams propagate in EIT windows ${ }^{47}$, or ones which rely on the strong interaction between Rydberg atoms ${ }^{31-34}$. Once in this regime, it will be possible to experimentally address the questions $s^{38,39}$ related to the effects of back-action noise on the signal.

\section{Methods}

Methods and any associated references are available in the online version of the paper.

Received 19 December 2014; accepted 13 July 2015; published online 24 August 2015

\section{References}

1. Schmidt, H. \& Imamoglu, A. Giant Kerr nonlinearities obtained by electromagnetically induced transparency. Opt. Lett. 21, 1936-1938 (1996)

2. Harris, S. E. \& Hau, L. V. Nonlinear optics at low light levels. Phys. Rev. Lett. 82, 4611-4614 (1999).

3. Harris, S. E. \& Yamamoto, Y. Photon switching by quantum interference. Phys. Rev. Lett. 81, 3611-3614 (1998).

4. Deutsch, I. H., Chiao, R. Y. \& Garrison, J. C. Diphotons in a nonlinear Fabry-Perot resonator: Bound states of interacting photons in an optical quantum wire. Phys. Rev. Lett. 69, 3627-3630 (1992).

5. Milburn, G. Quantum optical Fredkin gate. Phys. Rev. Lett. 62, 2124-2127 (1989).

6. Nemoto, K. \& Munro, W. J. Nearly deterministic linear optical controlled-NOT gate. Phys. Rev. Lett. 93, 250502 (2004).

7. Fleischhauer, M., Imamoglu, A. \& Marangos, J. Electromagnetically induced transparency: Optics in coherent media. Rev. Mod. Phys. 77, 633-673 (2005).

8. Glauber, R. J. Coherent and incoherent states of the radiation field. Phys. Rev. 131, 2766-2788 (1963)

9. Glauber, R. J. The quantum theory of optical coherence. Phys. Rev. 130, 2529-2539 (1963).

10. Firstenberg, O. et al. Attractive photons in a quantum nonlinear medium. Nature 502, 71-75 (2013).

11. Imoto, N., Haus, H. A. \& Yamamoto, Y. Quantum nondemolition measurement of the photon number via the optical Kerr effect. Phys. Rev. A 32, 2287-2292 (1985).
12. Braginsky, V. B., Vorontsov, Y. I. \& Thorne, K. S. Quantum nondemolition measurements. Science 209, 547-557 (1980).

13. Grangier, P., Levenson, J. A. \& Poizat, J.-P. Quantum non-demolition measurements in optics. Nature 396, 537-542 (1998).

14. Vitali, D., Fortunato, M. \& Tombesi, P. Complete quantum teleportation with a Kerr nonlinearity. Phys. Rev. Lett. 85, 445-448 (2000).

15. Turchette, Q. A., Hood, C. J., Lange, W., Mabuchi, H. \& Kimble, H. J. Measurement of conditional phase shifts for quantum logic. Phys. Rev. Lett. 75, 4710-4713 (1995)

16. Raimond, J.-M., Brune, M. \& Haroche, S. Manipulating quantum entanglement with atoms and photons in a cavity. Rev. Mod. Phys. 73, 565-582 (2001).

17. Guerlin, C. et al. Progressive field-state collapse and quantum non-demolition photon counting. Nature 448, 889-893 (2007).

18. Gleyzes, S. et al. Quantum jumps of light recording the birth and death of a photon in a cavity. Nature 446, 297-300 (2007).

19. Rauschenbeutel, A. et al. Step-by-step engineered multiparticle entanglement. Science 288, 2024-2028 (2000).

20. Wallraff, A. et al. Strong coupling of a single photon to a superconducting qubit using circuit quantum electrodynamics. Nature 431, 162-167 (2004).

21. Devoret, M. \& Schoelkopf, R. Superconducting circuits for quantum information: An outlook. Science 339, 1169-1174 (2013).

22. Matsuda, N., Shimizu, R., Mitsumori, Y., Kosaka, H. \& Edamatsu, K. Observation of optical-fibre Kerr nonlinearity at the single-photon level Nature Photon. 3, 95-98 (2009).

23. Venkataraman, V., Saha, K. \& Gaeta, A. L. Phase modulation at the few-photon level for weak-nonlinearity-based quantum computing. Nature Photon. 7, 138-141 (2013).

24. O'Shea, D., Junge, C., Volz, J. \& Rauschenbeutel, A. Fiber-optical switch controlled by a single atom. Phys. Rev. Lett. 111, 193601 (2013).

25. Hendrickson, S., Pittman, T. \& Franson, J. Nonlinear transmission through a tapered fiber in rubidium vapor. J. Opt. Soc. Am. B 26, 267-271 (2009).

26. Spillane, S. et al. Observation of nonlinear optical interactions of ultralow levels of light in a tapered optical nanofiber embedded in a hot rubidium vapor. Phys. Rev. Lett. 100, 233602 (2008).

27. Volz, J., Scheucher, M., Junge, C. \& Rauschenbeutel, A. Nonlinear $\pi$ phase shift for single fibre-guided photons interacting with a single resonator-enhanced atom. Nature Photon. 8, 965-970 (2014).

28. Urban, E. et al. Observation of Rydberg blockade between two atoms. Nature Phys. 5, 110-114 (2009)

29. Pritchard, J. D. et al. Cooperative atom-light interaction in a blockaded Rydberg ensemble. Phys. Rev. Lett. 105, 193603 (2010).

30. Saffman, M., Walker, T. G. \& Mølmer, K. Quantum information with Rydberg atoms. Rev. Mod. Phys. 82, 2313-2363 (2010).

31. Parigi, V. et al. Observation and measurement of interaction-induced dispersive optical nonlinearities in an ensemble of cold Rydberg atoms. Phys. Rev. Lett. 109, 233602 (2012).

32. Peyronel, T. et al. Quantum nonlinear optics with single photons enabled by strongly interacting atoms. Nature 488, 57-60 (2012).

33. Chen, W. et al. All-optical switch and transistor gated by one stored photon. Science 341, 768-770 (2013).

34. Baur, S., Tiarks, D., Rempe, G. \& Dürr, S. Single-photon switch based on Rydberg blockade. Phys. Rev. Lett. 112, 073901 (2014).

35. Gorniaczyk, H., Tresp, C., Schmidt, J., Fedder, H. \& Hofferberth, S. Single-photon transistor mediated by interstate Rydberg interactions. Phys. Rev. Lett. 113, 053601 (2014).

36. Guerreiro, T. et al. Nonlinear interaction between single photons. Phys. Rev. Lett. 113, 173601 (2014).

37. Shapiro, J. H. Single-photon Kerr nonlinearities do not help quantum computation. Phys. Rev. A 73, 062305 (2006).

38. Shapiro, J. H. \& Razavi, M. Continuous-time cross-phase modulation and quantum computation. New J. Phys. 9, 16 (2007).

39. Gea-Banacloche, J. Impossibility of large phase shifts via the giant Kerr effect with single-photon wave packets. Phys. Rev. A 81, 043823 (2010).

40. Munro, W. J., Nemoto, K. \& Spiller, T. P. Weak nonlinearities: A new route to optical quantum computation. New J. Phys. 7, 137 (2005).

41. Lo, H.-Y. et al. Electromagnetically-induced-transparency-based cross-phase-modulation at attojoule levels. Phys. Rev. A 83, 041804 (2011)

42. Smith, W. P., Reiner, J. E., Orozco, L. A., Kuhr, S. \& Wiseman, H. M. Capture and release of a conditional state of a cavity QED system by quantum feedback. Phys. Rev. Lett. 89, 133601 (2002).

43. Foster, G. T., Smith, W. P., Reiner, J. E. \& Orozco, L. A. Third-order correlations in cavity quantum electrodynamics. J. Opt. B 4, S281 (2002).

44. Foster, G. T., Smith, W. P., Reiner, J. E. \& Orozco, L. A. Time-dependent electric field fluctuations at the subphoton level. Phys. Rev. A 66, 033807 (2002).

45. Feizpour, A., Dmochowski, G. \& Steinberg, A. M. Short-pulse cross-phase modulation in an electromagnetically-induced-transparency medium. Preprint at http://arXiv.org/abs/1406.0245 (2014). 
46. Wolfgramm, F. et al. Bright filter-free source of indistinguishable photon pairs. Opt. Express 16, 18145-18151 (2008).

47. Wang, Z.-B., Marzlin, K.-P. \& Sanders, B. C. Large cross-phase modulation between slow copropagating weak pulses in $\mathrm{Rb}^{87}$. Phys. Rev. Lett. 97, 063901 (2006).

\section{Acknowledgements}

This work was funded by NSERC, CIFAR and QuantumWorks. We would like to thank A. Hayat for useful discussions, and A. Stummer for designing and building several electronic devices for this experiment.

\section{Author contributions}

All authors have contributed to the design of the experiment, interpretation of the results, and preparation and revisions of the manuscript.

\section{Additional information}

Supplementary information is available in the online version of the paper. Reprints and permissions information is available online at www.nature.com/reprints.

Correspondence and requests for materials should be addressed to A.F.

\section{Competing financial interests}

The authors declare no competing financial interests. 


\section{Methods}

Atom preparation. The atoms are prepared in a magneto-optical trap using three beams and their retro-reflections along with a magnetic field gradient of $20 \mathrm{G} \mathrm{cm}^{-}$ on the axis of the quadrupole coil. Each of the three beams has a 'trapping' $(2.5 \mathrm{~cm}$ diameter) and a 'repumper' ( $1 \mathrm{~cm}$ diameter) component. The trapping beam is tuned to $20 \mathrm{MHz}$ below the cycling transition $F=3 \rightarrow F^{\prime}=4$ and the repumper is tuned close to resonance on the $F=2 \rightarrow F^{\prime}=3$ transition to keep the population in the trapping ground state. The beams and the magnetic field gradient are turned of every $22 \mathrm{~ms}$, leaving the atoms to freely expand and allowing them to be probed for $1.5 \mathrm{~ms}$, as explained in Supplementary Fig. 4.

Probe phase measurement. The nonlinear effect we are interested in is a change in the probe refractive index, which is linear in the signal photon number and manifests itself as a phase shift of the probe field. To measure this probe phase shift, frequency-domain interferometry is used ${ }^{48,49}$. The probe beam is comprised of two different frequency components, which co-propagate through the atomic cloud. One frequency component is tuned on resonance with the $F=2 \rightarrow F^{\prime}=3$ transition, whereas the other is $+100 \mathrm{MHz}$ detuned, serving as a phase reference; any phase or amplitude change due to the atoms appears as a phase or amplitude change of the resulting $100 \mathrm{MHz}$ beating signal. The probe beam is detected on a fast avalanche photodiode; demodulation of the resultant electrical signal at $100 \mathrm{MHz}$ allows us to extract the phase and amplitude change experienced by the on-resonance probe field. The analysis bandwidth for the demodulation is $2 \mathrm{MHz}$, which is matched to the EIT window for optimum signal-to-noise ratio. A stable $10 \mathrm{MHz}$ clock is used to generate a $100 \mathrm{MHz}$ signal to act as the reference for the modulation and demodulation. An important advantage of using frequencydomain interferometry and demodulation is that the measurement is insensitive to any source of variation that does not have a component at $100 \mathrm{MHz}$.

Although the signal pulses are only 40 or $100 \mathrm{~ns}$ in duration, the XPS lasts for approximately $300 \mathrm{~ns}$, as determined by the response time of the EIT medium. We have detailed this transient behaviour in refs 45,50. Precise timing of the signal pulses with respect to our measurement cycle trigger allows us to locate the XPS signal. As a phase reference, we measure the probe phase in $200 \mathrm{~ns}$ windows directly before and after the passage of the signal pulse. The XPS is then defined as the difference between the average probe phase over the $300 \mathrm{~ns}$ effect and the averaged reference phase.

Probe phase noise. We measure a single-shot phase uncertainty of $50 \mathrm{mrad}$ (there are approximately 18,000 probe photons per measurement window before the interaction, one sixth of which reach the detector and give rise to a shot noise of $26 \mathrm{mrad}$ ). The extra noise in this experiment is due to electrical, digitizer and other technical noise sources. To reach the $\mu$ rad-level phase resolution we averaged over roughly 100 million shots; see 'Data collection' section for more details.

Probe and coupling fields. Our master laser is locked $30 \mathrm{MHz}$ below the $F=2 \rightarrow F^{\prime}=3$ transition of the D2 line in ${ }^{85} \mathrm{Rb}$. A portion of this light is frequency-shifted using an acousto-optic modulator (AOM) driven at $+130 \mathrm{MHz}$ to produce the off-resonance component of the probe, which is then passed through a second $\mathrm{AOM}$ driven at $-100 \mathrm{MHz}$ to create the on-resonance component. These two components are then combined on a beamsplitter, forming the probe beat signal. Another portion of the master laser is modulated using an electro-optic modulator (EOM) driven at $3 \mathrm{GHz}$. This modulated light is used as the seed for injection-locking a diode laser; locking to the lower side band of the modulation produces a coupling beam that is phase-locked to the probe, a necessary condition for establishing EIT. This coupling beam passes through a third $\mathrm{AOM}$, which serves to frequency shift it onto resonance with the $F=3 \rightarrow F^{\prime}=3$ transition, as well as to shutter the coupling light on and off in sync with the atomic duty cycle. The width of the EIT feature is linear in the coupling intensity and is measured to be $2 \mathrm{MHz}$ for all the data taken for this paper. The major source of ground state dephasing in our set-up is a Doppler broadening which is estimated to be around $300 \mathrm{kHz}$. Owing to this dephasing process, the optical density as seen by the probe beam in the presence of the on-resonance coupling is $0.7 \pm 0.1$. The optical density is defined as $\mathrm{OD}=-\ln \left(P_{\text {transmitted }}\left(\Delta_{p}\right) / P_{\text {incident }}\right)$, where $P$ is the optical power of the probe beam. $P_{\text {incident }}$ is the transmitted power in the absence of atoms and $P_{\text {transmitted }}$ is the transmitted power when the probe passes through the atoms; $\Delta_{p}$ is the detuning of the probe. Given the slope of the EIT feature in our experiment we estimate $c / v_{\mathrm{g}}=1 \times 10^{5}$, where $v_{\mathrm{g}}$ is the probe group velocity. We use $\pi$ and $\sigma^{+}$polarizations for the coupling and probe fields, respectively.

The powers of the probe components are $15 \mathrm{nW}$ on resonance and $180 \mathrm{nW}$ off resonance, and the coupling power is $20 \mu \mathrm{W}$.

Signal pulses. A portion of the injection-locked laser light is split off to produce the signal pulses. An AOM is used to frequency shift the light close to the $F=3 \rightarrow F^{\prime}=4$ transition as well as to create short pulses ( $40 \mathrm{~ns}$ or $100 \mathrm{~ns}$ full-width at half-maximum). The temporal profiles of the signal pulses are measured on an avalanche photodiode. By integrating the pulse power over time, the average energy, and therefore the average photon number, of each signal pulse can be measured. The polarization of the signal light is $\sigma^{+}$

Single-photon detection. The signal pulses are collected into a multimode fibre and detected on an SPCM. Other than the signal photons, any stray light which leaks into the signal collection can result in clicks. We use gate signals with durations matching the signal pulses to reject background photons. The residual probability of background counts is $13 \%$ for $100 \mathrm{~ns}$ pulses and $6 \%$ for $40 \mathrm{~ns}$ signal pulses. Most of these result from the near-resonant scattering of the probe and coupling beams from the atoms. The overall collection and detection efficiency is around $20 \%$, and for higher incident photon numbers more attenuation is added to keep the total click rate around $20-30 \%$. The details of the tagging procedure are explained in the Supplementary Information.

To reduce the background photon rate for cases of incident average photon number of 0.5 and 1 , the signal pulse duration was chosen to be $40 \mathrm{~ns}$, whereas it had been $100 \mathrm{~ns}$ for the data taken for higher signal power. One might expect this change to make the signal pulses more intense, and therefore make the nonlinear phase shift more than twice as large. However, we have previously shown ${ }^{45,50}$ that because of the bandwidth mismatch between the EIT window ( $2 \mathrm{MHz}$ here) and the signal pulses, the peak phase shift nearly saturates, and we expect an enhancement of only 1.5. Therefore, given the size of our phase measurement uncertainty, the two values are expected not to be statistically different.

The inferred average photon number difference between click and no-click cases is slightly different from unity owing to the effects of background counts, multiple-photon events and detection efficiency. To calculate the nonlinear phase shift per post-selected single photon, the measured phase differences are divided by the values of the inferred average photon number difference.

Data collection. For each data point, we took approximately 300 million shots over $14 \mathrm{~h}, 90$ million of which resulted in clicks at the SPCM. Because of our tagging procedure 90 million shots were discarded, and out of the remaining shots we observed 60 million click events and 150 million no-click events.

Focus size. The waists of the probe and signal beams inside the cloud are $13 \pm 1 \mu \mathrm{m}$, corresponding to a (two-sided) Rayleigh range of roughly $1.4 \mathrm{~mm}$. The choice of this focus size is to ensure that the Rayleigh range of the beams matches the size of the cloud. Focusing the beams tighter than the size of the cloud would be detrimental because it would reduce the interaction length and increase the probe phase shot noise (the intensity of the probe is limited and fixed by the saturation intensity of atoms). Also, focusing the beams any less tightly would produce a smaller intensity for given signal pulse energy, thereby decreasing the size of the nonlinear effect. The coupling beam waist is $0.5 \mathrm{~mm}$.

\section{References}

48. Chen, Y.-F., Liu, Y.-C., Tsai, Z.-H., Wang, S.-H. \& Ite, A. Y. Beat-note interferometer for direct phase measurement of photonic information. Phys. Rev. A 72, 033812 (2005).

49. Lo, H.-Y., Su, P.-C., Cheng, Y.-W., Wu, P.-I. \& Chen, Y.-F. Femtowatt-light-level phase measurement of slow light pulses via beat-note interferometer. Opt. Express 18, 18498-18505 (2010).

50. Dmochowski, G. et al. Observation of EIT-enhanced cross-phase modulation in the short-pulse regime. Preprint at http://arXiv.org/abs/1506.07051 (2015). 